Research article

\title{
Raltitrexed (Tomudex) administration in patients with relapsed metastatic colorectal cancer after weekly irinotecan/5-Fluorouracil/Leucovorin chemotherapy Nicolas Tsavaris ${ }^{1}$, Christos Kosmas ${ }^{2}$, Maria Vadiaka ${ }^{1}$ and Christos Koufos*1
}

Address: ${ }^{1}$ Medical Oncology Unit, Department of Pathophysiology, Laikon General Hospital, Athens University School of Medicine, Athens, Greece and ${ }^{2}$ Medical Oncology Unit, Department of Medicine, Helena-Venizelou Hospital, Athens, Greece

E-mail: Nicolas Tsavaris - tsavari1@otenet.gr; Christos Kosmas - ckosm@ath.forthnet.gr; Maria Vadiaka - micbcar@otenet.gr; Christos Koufos* - ckoufos@cc.uoa.gr

${ }^{*}$ Corresponding author

Published: 30 January 2002

BMC Cancer 2002, 2:2
Received: 17 December 200 I

Accepted: 30 January 2002

This article is available from: http://www.biomedcentral.com/I47I-2407/2/2

(c) 2002 Tsavaris et al; licensee BioMed Central Ltd. Verbatim copying and redistribution of this article are permitted in any medium for any purpose, provided this notice is preserved along with the article's original URL.

\begin{abstract}
Purpose: The present study aimed at evaluating the efficacy of Raltitrexed, a specific thymidilate synthase inhibitor, in patients with advanced colorectal cancer (ACC) in relapse ( $>8$ weeks) after a prior response or disease stabilization to first-line chemotherapy combination with Irinotecan+5Fluorouracil (5-FU)+Leucovorin (LV).

Methods: Twenty-five patients with metastatic ACC entered; 17 males $/ 8$ females, median age 61 (range: 47-70), median Karnovsky PS: 80 (70-90), and sites of metastases; liver: 2I, lung: 4, lymph nodes: 7, peritoneal: 5 and a life expectancy of at least 3 months, were entered in the present pilot study. All patients had progressed after prior chemotherapy with Irinotecan+5-FU+LV. Raltitrexed was administered at a dose of $3 \mathrm{mg} / \mathrm{m}^{2}$ i.v. every 21 days.

Results: Three patients (I2\%) achieved a partial response (PR), 8 (32\%) had stable disease (SD), and the remaining 14 (56\%) developed progressive disease (PD). Median time-to-progression (TTP) was 5.5 months (range, 2-8.5), and median overall survival (OS) 8 months (range, 4.0-12.5). Toxicity was generally mild; it consisted mainly of myelosuppression; neutropenia grade $\mathrm{I}-2: 52 \%-$ grade 3: $28 \%$, and anemia grade I-2 only: $36 \%$. Mild mucositis grade I-2 occured in $13.5 \%$ of patients and was the principal non-hematologic toxicity.

Conclusion: Response to treatment with Raltitrexed is limited in patients with ACC failing after an initial response or non-progression to the weekly Irinotecan+5-FU+LV combination. However, it appears that a limited number of patients with $P R / S D$ may derive clinical benefit, but final proof would require a randomized study.
\end{abstract}

\section{Background}

Treatment of advanced colorectal cancer has been minimally successful due to the poor response of the disease to classic cytotoxic agents. Antimetabolites, such as MTX and 5-FU, have been in clinical use for many years. Both agents exert their cytotoxic action by inhibiting thymidilate synthase (TS) the rate-limiting enzyme that methylates deoxyuridine monophosphate (dUMP) to thymidine monophosphate (TMP); the reaction requires reduced folate as a cofactor and leads to incorporation of thymi- 
dine triphosphate into the DNA [1]. 5-FU is converted intracellularly to 5-FdUMP, which inhibits TS. Folinic acid (leucovorin) potentiates this inhibitory effect on TS by forming a ternary complex with the enzyme. Moreover, 5FdUMP inhibits purine synthesis and exerts inhibitory effects not only on DNA but on RNA, as well. These nonspecific non-TS dependent effects on RNA are believed to account at a certain degree for the toxicity encountered with 5-FU, such as mucositis [1].

Raltitrexed (Tomudex) represents a specific TS inhibitor not requiring modulation and not having any non-specific effects on RNA. Phase II studies with Raltitrexed at 3 $\mathrm{mg} / \mathrm{m}^{2}$ iv every 21 days demonstrated activity in a variety of advanced solid tumors, and most notably in advanced colorectal cancer and breast cancer [2]. Moreover, a subsequent randomized trial comparing Raltitrexed versus 5$\mathrm{FU}+\mathrm{LV}$ in chemotherapy-naive patients with advanced colorectal cancer, demonstrated equal activity and survival figures with reduced toxicity, regarding mucositis and leukopenia, for Raltitrexed [3]. Response rates with Raltitrexed have been in the range of $20-30 \%$ in patients with advanced colorectal cancer $[2,3]$.

Irinotecan represents an active agent in advanced colorectal cancer relapsing after 5-FU+LV based combination as demonstrated in two recent large multi-institutional controlled phase III studies $[4,5]$. However, despite the clinical benefit derived from CPT-11 treatment in relapsed ACC, patients generally develop PD quite rapidly and might be candidates for further experimental treatment. Sometimes long response durations are observed. Furthermore, as demonstrated in two recent randomized trials by Douillard et al[6] and Saltz et al[7], combination chemotherapy with 5-FU, LV and Irinotecan provided improved response rates and survival advantage over both bolus 5FU and continuous infusion 5-FU modulated with LV without compromising quality of life [7]. These results are very encouraging and suggest that the addition of Irinotecan to $\mathrm{LV}+5-\mathrm{FU}$ has an important role in the front-line treatment of patients with ACC.

It is currently unknown whether treatment with Raltitrexed after prior lrinotecan+5-FU+LV would have any clinical effect, since both Raltitrexed and 5-FU target the same enzyme (TS) and it is therefore anticipated that a high level of cross-resistance might exist. Moreover, lrinotecan $+5-\mathrm{FU}+\mathrm{LV}$ is currently the most active first-line and it is not yet known whether other second-line drugs might be active in this setting.

\section{Patients and Methods Patients}

Twenty-five patients with reccurent or metastatic adenocarcinoma of the colon and rectum, that had been treated
Table I: Patients' characteristics

\begin{tabular}{ll}
\hline Number & 25 \\
Sex & \\
$\quad$ Men & 17 \\
Women & 8 \\
Median Age & 61 (range, 47-70) \\
Median PS (Karnovsky) & 80 (range, 70-90) \\
Primary & \\
$\quad$ Colon & 21 \\
Rectum & 4 \\
Metastases & \\
Liver & 21 \\
Lung & 4 \\
$\quad$ Lymph nodes & 7 \\
Peritoneal & 5 \\
\end{tabular}

at first-line with lrinotecan $+5-\mathrm{FU}+\mathrm{LV}$ and relapsed at least 8 weeks after last treatment entered this study (Table 1 ).

\section{Eligibility criteria}

Eligibility criteria included bi-dimensionally measurable disease, performance status (PS) (Karnofsky) equal or $>70$, life expectancy of at least 3 months. Patients had to have normal hematologic, renal or hepatic function tests unless the abnormalities had resulted from direct tumor invasion. Moreover, absence of brain metastases, active ischemic cardiac disease or cardiac insufficiency, absence of psychotic disorders, diabetes and cirrhosis was required. A histological documentation of measurable metastatic disease was obtained whenever possible. All patients had previously undergone chemotherapy with the weekly Irinotecan $\left(60 \mathrm{mg} / \mathrm{m}^{2}\right)+5$-FU $(400 \mathrm{mg} /$ $\left.\mathrm{m}^{2}\right)+\mathrm{LV}\left(200 \mathrm{mg} / \mathrm{m}^{2}\right.$ ) regimen (with no planned breaks unless the occurrence of grade III toxicity or PD) and had achieved a CR/PR or SD and progressed at least 8 weeks off treatment. Informed consent was obtained from all patients according to Institutional policies.

\section{Treatment}

Treatment was carried-out in the day clinic. Raltitrexed was administered at a dose of $3 \mathrm{mg} / \mathrm{m}^{2}$ i.v. infusion for 30 min, every 21 days. Treatment was continued for 2 cycles beyond maximal response (CR/PR) or until tumor progression. In the event of > grade II myelosupppression on the day of treatment, or other non-hematologic toxicities according to WHO [8], treatment was delayed until recovery, or for a maximum of 2 weeks, after which no further treatment was administered in the case that blood counts had not returned to normal (neutrophils $>1500 / \mu \mathrm{L}$ and platelets $>100.000 / \mu \mathrm{L}$ ). The dose of Raltitrexed was kept stable, if no toxicity $\geq$ grade 3 was encountered. In the case of $\geq$ grade 3 hematologic or non-hematologic (except alopecia, nausea and vomiting) toxicity subsequent doses of 
Raltitrexed were reduced by $20 \%$, and beyond a $40 \%$ maximum dose reduction no further treatment was administered.

\section{Criteria for response and follow-up}

Before each treatment cycle every patient had a complete blood count, serum biochemistry, ECG, chest roentgenography, and abdominal CT scan. Between the treatment cycles CBC's were performed weekly. Patients were evaluated for response every two cycles of treatment. A complete response (CR) was defined as a complete dissappearance of all clinically evident disease. Partial response (PR) was defined as a decrease of than $50 \%$ in the sum of the products of the largest perpedicular diameters of the measurable lesions. Both CR and PR had to have a minimum duration of 4 weeks. A $<50 \%$ reduction up to $25 \%$ increase in the sum of the products of the largest perpendicular diameters of all measurable lesions defined as Stable Diasease (SD). For all response definitions a minimum 4-week duration was required for qualifying for each type of response (CR, PR, SD). Progressive Disease (PD) was an increase of the above measurements or the appearance of new lesions. Clinical benefit was defined as improvement of disease-related symptoms, an increase in Karnovsky PS by $>15$, and the absence of major cumulative toxicity, such as fatigue or weight loss related to treatment.

After the end of treatment, patients were followed with clinical examination, blood counts, serum biochemistry and serum tumor marker levels (CEA, CA-19.9), CT scans of the chest and abdomen or any other indicated sites every 3 months.

\section{Toxicity}

Toxicity was estimated according to WHO criteria [8]. We also evaluated the highest grade of toxicity for each patient during the treatment course, in order to find during which time period of therapy we would expect the highest grade of toxicity.

\section{Statistical methods}

Time to progression was calculated from the day of study entry until evidence of progressive disease; overall survival was measured from the day of entry to the study until last folow-up or death. The $95 \%$ confidence intervals $(\mathrm{Cl})$ for response rates were calculated from the binomial distribution. For a new drug in the setting of relapsed ACC, a 15\% response rate $(\mathrm{RR} ; \mathrm{CR}+\mathrm{PR})$ was anticipated as satisfactory to proceed to further phase II patient accrual. With a maximum of $7 \%$ standard error (SE) for RR, the minimum sample size required to satisfy these conditions was $25 \mathrm{pa}-$ tients. If less than 4 responses were recorded among the initial 25 patients the drug would be considered inactive (with a power $>90 \%$, at a 0.05 significance) and the study would be stopped.

\section{Results}

Twenty-five patients entered the study between September 1999 and October 2000 and all were evaluable for toxicity and response. Three patients experienced achieved PR, 8 (32\%) had SD, and the remaining 14 (56\%) developed PD. All three patients with PR had a prior response (1 CR and 2 PRs) to first-line weekly lrinotecan+5-FU+LV. Moreover, $7 / 8$ patients with SD to second-line Raltitrexed had experienced a PR to prior first-line lrinotecan+5-FU+LV. Indeed all 3 patients with a PR and 5/8 with SD to Raltitrexed had improvement in disease-related symptoms (overall 8/25 patients; 32\%), while 2 of these patients had improvement of PS from 70 to 90 , in addition, and were therefore considered as having derived clinical benefit according to our definitions (see also Patients \& Methods). In view of the lower than the pre-determined RR (see Statistical Methods) for further patient accrual, we decided to terminate the study at this stage.

Median TTP was 5.5 mo (range, 2-8.5), and median OS 8 mo (range, 4.0-12.5). The median duration of previous treatment with lrinotecan $+5-\mathrm{FU}+\mathrm{LV}$ first-line chemotherapy was 5 mo (range, $4-8$ ), corresponding to 5 treatment cycles (range, 4-8) (1 treatment cycle with lrinotecan +5 $\mathrm{FU}+\mathrm{LV}=1 \mathrm{mo} ; 4$ weeks). After a median time of $4.5 \mathrm{mo}$ (range, 2-14) off treatment all patients started secondline chemotherapy with Raltitrexed for a median duration of treatment of 3 mo (range 1-7). The median number of Raltitrexed cycles was 4 (range, 2-8). For the three patients achieving PR, TTPs were 3.5, 6.5 and $8.5 \mathrm{mo}$, while survivals were $6,8.5$, and 12.5 mo, respectively.

Toxicity was evaluated according to WHO grading and consisted principaly of myelotoxicity; neutropenia (Grade 1-2, 54/104 cycles: 52\%; Grade 3, 29/104 cycles: 28\%), anemia (decrease of $\mathrm{Hgb}>3 \mathrm{gr} / \mathrm{dl}$ ) grade $1-2$ only (37/ 104 cycles; 36\%), and diarrhea Grade 1-2, 32/104 cycles: 31\%, Grade 3, 26/104 cycles: 25\%. Other toxicity parameters include nausea-vomiting (Grade 1-2, 33/104 cycles; $32 \%$ ), mucositis (Grade 1-2, 14/104 cycles; $13.5 \%$ ), fatigue ( $11 / 25$ patients; $44 \%)$, worsening PS ( $6 / 25$ patients; $24 \%)$, transaminase increase (3/25 patients, $12 \%)$.

\section{Discussion}

The present study demonstrated that the activity of Raltitrexed when used as salvage treatment in ACC after relapse/progression with first-line weekly lrinotecan +5 $\mathrm{FU}+\mathrm{LV}$ is rather limited. However, a not negligible proportion of patients (32\%) derives clinical benefit. It should be noted that all these 3 patients achieving PR and $7 / 8$ with SD to Raltitrexed, i.e. overall $40 \%$ of patients, had responded to prior lrinotecan $+5-\mathrm{FU}+\mathrm{LV}$. 
Table 2: Toxicity parameters ( 104 cycles in 25 patients).

\begin{tabular}{|c|c|c|c|}
\hline Parameter & Grade (WHO) & Per Cycle & Percentage (\%) \\
\hline \multirow[t]{2}{*}{ Neutropenia } & $1-2$ & $54 / 104$ & $52 \%$ \\
\hline & $3-4$ & $29 / 104$ & $28 \%$ \\
\hline Anemia $(\mathrm{Hg}>3 \mathrm{gr} / \mathrm{dl})$ & $1-2$ & $37 / 104$ & $36 \%$ \\
\hline \multirow{2}{*}{ Diarrhea } & $1-2$ & $32 / 104$ & $31 \%$ \\
\hline & 3 & $26 / 104$ & $25 \%$ \\
\hline Nausea-Vomiting & $1-2$ & $33 / 104$ & $32 \%$ \\
\hline Mucositis & $\mathrm{I}-2$ & $14 / 104$ & $13.5 \%$ \\
\hline \multicolumn{4}{|c|}{ Per Patient } \\
\hline Fatigue & $\mathrm{I}-2$ & $11 / 25$ & $44 \%$ \\
\hline Transaminases (ALT/AST) & $\mathrm{I}-2$ & $3 / 25$ & $12 \%$ \\
\hline Worsening PS & & $6 / 25$ & $24 \%$ \\
\hline
\end{tabular}

The experience was different when Raltitrexed has been applied as a first-line agent. Three large multi-institutional randomized clinical trials demonstrated that the objective RR (CR+PR) with Raltitrexed ranged from $14 \%$ to $19 \%$, and was similar to that of 5-FU modulated by either lowdose or high-dose Leucovorin [9-11]. However, in one of the above studies [10] with a minimum follow-up time of 12 months, there was no significant difference between the groups in RR (14\% Raltitrexed vs 15\% 5-FU+LV), but median TTP and OS time were significantly longer for the 5-FU+LV group. Median survival time was 12.7 months and 9.7 months for the 5-FU+LV and Raltitrexed groups, respectively [10]. The number of patients with SD was also similar for Ratitrexed and 5-FU/LV. Moreover, survival was comparable between Raltitrexed and 5-FU+LV treated groups in both studies. It was concluded that Raltitrexed might be favored over either 5-FU+LV combinations, given its ease of administration (one day every 3 weeks), reduced visits in the outpatient department and overall length of treatment and reduced non-hematologic toxicities, such as mucositis, diarrhea and hematologic toxicity, predominantly mild and uncomplicated neutropenia.

Despite the fact that Raltitrexed produces palliative benefits for patients with advanced colorectal cancer when used as first-line treatment, it is questionable whether it has any significant impact when used as second or thirdline treatment in patients failing prior $5-\mathrm{FU}+\mathrm{LV}$ and CPT11-based regimens.

The present phase II study indicates that Raltitrexed has limited activity in patients that have failed the most active first-line chemotherapy regimen for ACC, incorporating weekly lrinotecan $+5-\mathrm{FU}+\mathrm{LV}[6,7]$. The possible explanations are that Raltitrexed and 5-FU have the same biochemical target; thymidylate synthase, however, with different properties of enzyme inhibition [10], or that pa- tients with advanced stages of disease and heavily pretreated might have limited chances to respond or benefit from any type of further treatment. Moreover, given that a prerequisite for entering the present study was that, patients had to have progressed from PR or SD at least after 2 months after the end of first-line treatment, it is anticipated that a group of patients with rather sensitive disease might have been selected. As the RR was only $12 \%$, the present study demonstrates that at relapse, after the most active at present first-line regimen-incorporating all most active agents-the emerging tumor cell clones are possibly resistant to any available further alternative treatment. However, a recent study in patients with ACC refractory to lrinotecan/5-FU/LV reported a 21\% response rate with infusional Oxaliplatin/5-FU/LV (Oxaliplatin-de Gramont regimen) [12].

Toxicity was acceptable in the present study, albeit with an increased incidence of neutropenia, anemia, nausea and diarrhea, most likely attributed to heavy pre-treatment and/or more advanced disease status and functional impairment. However, a high rate of unexpected toxicity has been reported with Raltitrexed with an $18 \%$ serious adverse event (SAE) rate and $4 \%$ toxic deaths compared to $3 \%$ SAE for the de Gramont regimen and $12 \%$ for the continuous low-dose 5-FU Lokich regimen, with no toxic deaths in the latter two 5-FU regimens in a multicenter randomized trial comparing the above three regimens $[13,14]$. Based on these findings, further interest in this agent has substantially declined.

\section{Conclusions}

The present study demonstrated that Raltitrexed when used as second-line treatment after prior lrinotecan +5 $\mathrm{FU}+\mathrm{LV}$ in ACC has limited activity, a $12 \% \mathrm{RR}$, and provides clinical benefit in $32 \%$ of these patients pre-treated with the most active first-line combination of lrinote- 
can $+5-F U+L V$. Its application in earlier phases of treatment and possibly in combination with other active agents, such as oxaliplatin in second-line might further improve the outcome of these patients.

\section{Competing interests}

None declared

\section{References}

I. Pinedo HM, Peters GF: Fluorouracil: biochemistry and pharmacology. J Clin Oncol 1988, 6:1653-1664

2. Cunningham D, Zalcberg J, Smith I, Gore M, Pazdur R, Burris H, Merpol NJ, Kennealy G, Seymour L, the "Tomudex" International Study Group: "Tomudex" (2DI694): a novel thymidylate synthase inhibitor with clinical antitumour activity in a range of solid tumours. Ann Oncol 1996, 7:179-182

3. Cunnningham D, Zalcberg JR, Rath U, Oliver I, Van Cutsem E, Svensson C, Seitz JF, Harper P, Kerr D, Perez-Manga G: Final results of a randomized trial comparing "Tomudex" (raltitrexed) with 5-Fluorouracil plus leucovorin in advanced colorectal cancer. Ann Oncol 1996, 7:961-965

4. Cunningham D, Pyrhonen S, James RD, Punt CJA, Hickish TF, Heikkila R, Johannesen TB, Starkhammar H, Tophan CA, Award L, et al: Randomized trial of irinotecan plus supportive care versus supportive care alone after fluorouracil failure for patients with metastatic colorectal cancer. Lancet 1998, 352:|4|3-|4|8

5. Rougier P, Van Cutsem E, Bajetta E, Niederle N, Possinger K, Labianca R, Navarro M, Morant R, Bleiberg H, Wils J, et al: Randomised trial of irinotecan versus fluorouracil by continuous infusion after fluorouracil failure in patients with metastatic colorectal cancer. Lancet 1998, 352:1407-1412

6. Douillard JY, Cunningham D, Roth AD, Navarro M, James RD, Karasek P, Jandik P, Iveson T, Carmichael J, Alakl M: Irinotecan combined with fluorouracil compared with fluorouracil alone as first line treatment for metastatic colorectal carcinoma: a multicenter randomize trial. Lancet 2000, 255:104I-1047

7. Saltz LB, Cox JV, Rosen LS, Fehrenbacher L, Moore MJ, Maroun JA, Ackland SP, Locker PK, Pirotta N, Elfring GL, Miller LL: Irinotecan plus fluorouracil and leucovorin for metastatic colorectal cancer. New Engl J Med 2000, 343:905-9|4

8. World Health Organization: Handbook for reporting results of cancer treatment no. 48. Geneva: World Health Organization, 1979

9. Cocconi G, Cunningham D, Van Cutsen E, Francois E, Gustavsson B, van Hazel G, Kerr D, Rossinger K, Hietschold SM, on behalf of the Tomudex Colorectal Cancer Study Group: Open, randomized, multicenter trial of raltitrexed versus fluorouracil plus highdose leucovorin in patients with advanced colorectal cancer. J Clin Oncol 1998, 16:2943-2952

10. Rustum YM, Harstrick A, Cao S, Vanhoefer U, Yin M-B, Wilke H, Seeber S: Thymidylate synthase inhibitors in cancer therapy: direct or indirect inhibitors. I Clin Oncol 1997, 15:389-400

II. Pazdur R, Vincent M: Raltitrexed (Tomudex) versus 5-Fluorouracil and Leucovorin (5-FU+LV) in patients with advanced colorectal cancer (ACC): Results of a randomized, multicenter, North American trial. Proc Am Soc Clin Oncol 1997, 16:228a

12. Tournigand C, Louvet C, Quinaux E, Andre T, Lledo G, Flesch M, Ganem G, Landi B, Colin P, Denet C, Mery-Mignard D, Risse M, Buyse $M$, de Gramont A: FOLFIRI followed by FOLFOX versus FOLFOX followed by FOLFIRI in metastatic colorectal cancer (MCRC): final results of a phase III study. Proc Am Soc Clin Oncol 200I, 20:124a

13. Maughan TS, James RD, Kerr D, McArdle C, Ledermann JA, Seymour M, Johnston C, Stephens RJ, on behalf of the British MRC Colorectal Cancer Working Party: Preliminary results of a randomized trial comparing 3 chemotherapy regiments (de Gramont, Lokich and Raltitrexed) in metastatic colorectal cancer. Proc Am Soc Clin Oncol 1999, 18:262a

14. Maughan T, James R, Kerr D, Ledermann J, McArdle C, Seymour M, Johnston $C$, Stephens R: Excess treatment related deaths and impaired quality of life show raltitrexed is inferior to infusional 5FU regimens in the palliative chemotherapy of ad- vanced colorectal cancer (CRC): Final results of MRC CR06. Ann Oncol 2000, I I:43
Publish with BioMed Central and every scientist can read your work free of charge

"BioMedcentral will be the most significant development for disseminating the results of biomedical research in our lifetime." Paul Nurse, Director-General, Imperial Cancer Research Fund

Publish with BMC and your research papers will be:

- available free of charge to the entire biomedical community

- peer reviewed and published immediately upon acceptance

- cited in PubMed and archived on PubMed Central

- yours - you keep the copyright

Submit your manuscript here:

http://www.biomedcentral.com/manuscript/
BioMedcentral.com editorial@biomedcentral.com 